\title{
EFEITOS DAS INTERAÇÕES SOBRE CINCO GALÁXIAS EM PARES OBTIDAS COM O GEMINI/GMOS-S: SÍNTESE DE POPULAÇÃO ESTELAR
}

\section{EFFECTS OF INTERACTIONS ON FIVE GALAXY PAIRS OBTAINED WITH GEMINI/GMOS-S: STELLAR POPULATION SYNTHESIS}

\author{
Deise Aparecida Rosa ${ }^{1}$ \\ Oli Luiz Dors Jr. ${ }^{2}$ \\ Ângela Cristina Krabbe ${ }^{2}$
}

RESUMO: Neste trabalho, apresenta-se um estudo sobre a influência das interações na população estelar em uma amostra de pares de galáxias. Foram utilizados dados de espectroscopia de fenda longa na região espectral 3000-7000 Å, obtida com o telescópio do Gemini Sul/GMOS, para uma amostra de cinco pares de galáxias. Esses dados foram combinados com modelos de síntese de populações estelares usando o programa STARLIGHT. Os resultados mostraram que alguns objetos são dominados por populações mais jovens, com idade até 500 bilhões de anos e outros têm um perfil ao longo do disco com população estelar composto de elementos heterogêneos. Conclui-se que as interações alteram, significativamente, a história da formação estelar de pares de galáxias.

Palavras-chave: interação de galáxia; síntese de população estelar.

ABSTRACT: This work presents a study about the influence of interactions on the stellar population in a sample of galaxy pairs. We used data from long slit spectroscopy in the spectral region 3000-7000 $\AA$ obtained with the GMOS/Gemini South telescope for a sample of five galaxy pairs. The data were combined with stellar population synthesis models using the program STARLIGHT. We found that some objects are dominated by younger populations with up to 500 billion years and others have a profile along the disk with stellar population composed of heterogeneous elements. We conclude that interactions significantly change the history of star formation of galaxy pairs.

Keywords: interaction galaxy; stellar population synthesis.

\footnotetext{
1 Doutoranda em Física e Astronomia - Universidade do Vale do Paraíba - Univap / Instituto de Pesquisa e Desenvolvimento IP\&D. E-mail: deiserosa@univap.br.

${ }^{2}$ Docente da Univap / IP\&D - Laboratório de Física e Astronomia. E-mails: olidors@hotmail.com; angela.krabbe@gmail.com.

Revista Univap - Edição Especial - revista.univap.br 


\section{INTRODUÇÃO}

Estudos de processos físicos presentes em interação e fusão de galáxias no universo local são de fundamental importância para entender a história de formação da população estelar e a evolução química desses objetos. Esses processos desencadeiam um amplo conjunto de fenômenos físicos e morfológicos, tais como, longas caudas compostas de gás, poeira e estrelas, assim como, núcleos de matéria desacoplados cinematicamente da galáxia hospedeira (STRUCK, 1999). Essas características são consideradas como evidências que interações são mecanismos que induzem uma alta taxa de formação estelar ao longo do disco das galáxias envolvidas nesse processo (BUSHOUSE, 1986; KENNICUTT et al., 1987; DONZELLI; PASTORIZA, 1997, FERREIRO; PASTORIZA, 2004). Esse aumento ocorre devido às perturbações dos discos galácticos por efeito de maré que originam fluxos de gás em direção às regiões centrais galácticas, fornecendo, assim, combustível para formar estrelas com altas taxas
(BARNES; HERNQUIST, 1996; IONO; YUN; MIHOS, 2004; SPRINGEL, 2005).

O movimento de gás, criado em ampla escala pela interação, aumenta a taxa de formação estelar e fluxo de gás, produzindo excitação do gás por choque nas regiões de formação de estrelas (VEILLEUX; CECIL; BLAND-HAWTHORN, 2005), tal como mostrado em estudos recentes de Galáxias Luminosas no Infravermelho (SOTO et al., 2012; RICH; KEWLEY; DOPITA, 2011; RICH et al., 2012).

Outrossim, o estudo de populações estelares estabelece uma relação direta com a evolução química das galáxias. Quando as estrelas estão nos seus estágios finais evolutivos, elas expelem suas camadas externas, proporcionando um enriquecimento do gás interestelar com seus elementos pesados, alterando, assim, a composição química do meio interestelar. Dessa forma, populações mais jovens de estrelas nascem a partir de um gás interestelar mais enriquecido com elementos pesados.

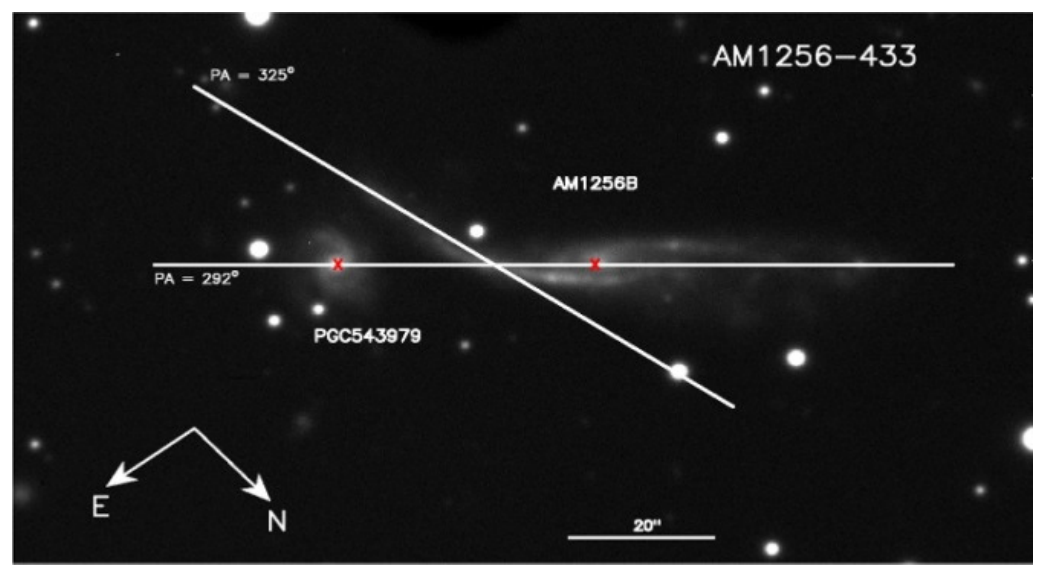

Figura 1 - Posições de fenda observada ao longo do raio de AM1256-433. 
Este trabalho tem por objetivo apresentar um estudo observacional para cinco sistemas de galáxias em pares (AM1054-325, AM1256-433, AM2030-303, AM2058-381 e AM 2229-735), um dos objetos é mostrado na Figura 1. Dessa forma, a partir do estudo das propriedades das populações estelares, poderemos compreender os efeitos causados pela interação.

\section{METODOLOGIA}

Neste trabalho, utilizamos o código de síntese espectral STARLIGHT, desenvolvido por Cid Fernandes et al., (2004/2005), baseado em técnicas computacionais originalmente desenvolvidas para síntese de população empírica e com alguns ingredientes adicionais de modelos de síntese evolutiva, com o propósito de obter as propriedades da história de formação das populações estelares, a partir dos espectros observados das galáxias em interação. O código STARLIGHT ajusta o espectro observado $O_{\lambda}$ de uma galáxia, utilizando uma combinação linear de $\mathrm{N} *$ elementos de uma base de populações estelares simples (SSPs), obtidas dos modelos de síntese evolutiva (BRUZUAL; CHARLOT, 2003). Por sua vez, esses modelos são baseados em bibliotecas de alta resolução de espectros observacionais, que permitem determinar a evolução espectral detalhada das SSPS através de um amplo intervalo de comprimento de onda de 3200-9500 $\AA$ e metalicidades. Esses modelos estelares Padova 1994, como proposto por Bruzual e Charlot (2003), foram utilizados uma função de massa inicial entre 0.1 e $100 M_{\odot}$ de Chabrier (2003). O software STARLIGHT modela a extinção devido à poeira interestelar, no qual se usa a lei de avermelhamento de Cardelli, Clayton e Mathis (1989) com $R v=3.1$, e parametrizado pela extinção na banda $\mathrm{V}, \mathrm{A}_{\mathrm{V}}$, de acordo com cada objeto de estudo e normalizado no $\lambda 5870 \AA$. Adotamos $N * 45$ bases de SSPs, totalizando quinze idades distintas de $\mathrm{t}=$ $0.001,0.003,0.005,0.01,0.025,0.04,0.1$, $0.3,0.6,0.9,1.4,2.5,5,11,13$ Gyr, e composta por três metalicidades, $Z=0.2 Z_{\odot}$, $1 Z_{\odot}$ e $2.5 Z_{\odot}$. Assumimos populações estelares de acordo com as contribuições de fluxo $(x)$ e massa $(m)$, em diferentes componentes, como:

$\left(x_{y}\right)$ - contribuição de população jovem com idade equivalente entre $1 \times 10^{8}<t \leq 5 \times 10^{8}$ anos, $\left(x_{i}\right)$ - população em idade intermediária em torno de $5 \times 10^{8}<\mathrm{t} \leq 2 \times 10^{9}$ anos e $\left(x_{0}\right)$ - população velha com idade $t$ $\geq 2 \times 10^{9}$ anos. 
Tabela 1- Resultado da contribuição da população estelar para a galáxia AM1256B ao longo das posições de fenda observada

\begin{tabular}{|c|c|c|c|c|c|c|c|c|c|c|c|c|c|c|}
\hline \multicolumn{15}{|c|}{$A M 1256-433$} \\
\hline \multicolumn{8}{|c|}{$P A=292^{\circ}$} & \multicolumn{7}{|c|}{$P A=325^{\circ}$} \\
\hline Posição & $x_{y}$ & $\mathbf{x}_{\mathbf{i}}$ & $\mathbf{X}_{0}$ & $\mathrm{~m}_{\mathrm{y}}$ & $\mathrm{m}_{\mathrm{i}}$ & $\mathrm{m}_{0}$ & $A_{v}$ & $x_{y}$ & $\mathbf{x}_{\mathbf{i}}$ & $\mathbf{X}_{0}$ & $m_{y}$ & $\mathrm{~m}_{\mathrm{i}}$ & $m_{0}$ & $A_{v}$ \\
\hline-18.795 & 84.6 & 14.9 & 0.9 & 9.3 & 45.9 & 44.9 & 0.94 & & & & & & & \\
\hline-6.041 & 0.0 & 78.3 & 23.5 & 0.0 & 62.2 & 37.8 & 0.00 & & & & & & & \\
\hline-4.699 & 0.0 & 103.5 & 0.0 & 0.0 & 100.0 & 0.0 & 0.12 & & & & & & & \\
\hline-4.027 & 7.1 & 95.3 & 0.0 & 0.2 & 99.8 & 0.0 & 0.00 & & & & & & & \\
\hline-3.356 & 14.6 & 88.8 & 0.0 & 0.4 & 99.6 & 0.0 & 0.41 & & & & & & & \\
\hline-2.685 & 25.3 & 43.1 & 37.3 & 0.4 & 33.5 & 66.1 & 0.45 & & & & & & & \\
\hline-1.342 & 14.2 & 62.1 & 26.6 & 0.7 & 31.5 & 67.8 & 0.44 & 23.6 & 73.2 & 5.7 & 2.5 & 37.8 & 59.7 & 0.10 \\
\hline-0.671 & 9.6 & 73.6 & 22.2 & 0.3 & 35.4 & 64.4 & 0.29 & 19.1 & 81.1 & 4.0 & 0.9 & 53.6 & 45.5 & 0.05 \\
\hline 0.000 & 14.4 & 45.2 & 44.8 & 0.8 & 21.8 & 77.4 & 0.60 & 15.5 & 78.2 & 9.7 & 0.4 & 50.8 & 48.8 & 0.10 \\
\hline 0.671 & 11.0 & 65.6 & 27.6 & 0.1 & 21.6 & 78.3 & 0.47 & 7.9 & 91.9 & 4.5 & 0.2 & 52.6 & 47.2 & 0.21 \\
\hline 1.342 & 7.2 & 88.7 & 7.8 & 0.1 & 46.2 & 53.7 & 0.42 & 19.1 & 73.0 & 12.8 & 0.4 & 37.6 & 62.0 & 0.40 \\
\hline 2.014 & 3.3 & 71.5 & 29.8 & 0.1 & 25.1 & 74.8 & 0.15 & 6.5 & 82.2 & 14.9 & 0.1 & 24.8 & 75.1 & 0.36 \\
\hline 2.685 & 20.6 & 59.6 & 22.9 & 0.8 & 33.9 & 65.3 & 0.52 & 7.7 & 89.5 & 8.6 & 0.2 & 32.7 & 67.1 & 0.68 \\
\hline 3.356 & 18.3 & 30.4 & 55.4 & 0.4 & 9.7 & 89.9 & 0.07 & 12.9 & 88.3 & 7.2 & 0.3 & 45.3 & 54.5 & 0.59 \\
\hline 4.027 & 5.5 & 89.7 & 10.5 & 0.2 & 36.5 & 63.3 & 0.36 & 9.5 & 88.9 & 4.8 & 0.9 & 48.1 & 51.0 & 0.48 \\
\hline 4.699 & 6.1 & 78.6 & 22.5 & 0.2 & 68.1 & 31.7 & 0.00 & 16.1 & 68.0 & 17.2 & 0.3 & 15.6 & 84.0 & 0.59 \\
\hline 5.370 & 13.2 & 33.2 & 60.0 & 0.3 & 19.7 & 80.0 & 0.35 & 28.4 & 60.1 & 16.8 & 1.1 & 16.2 & 82.7 & 0.50 \\
\hline 6.041 & 9.5 & 96.0 & 0.0 & 0.4 & 99.6 & 0.0 & 0.59 & 42.3 & 48.4 & 12.8 & 3.3 & 24.7 & 72.0 & 0.65 \\
\hline 6.712 & 9.7 & 57.8 & 39.3 & 0.2 & 51.8 & 48.0 & 0.07 & 47.0 & 32.4 & 18.7 & 0.8 & 9.3 & 89.8 & 0.33 \\
\hline 7.384 & 1.8 & 106.3 & 0.0 & 0.0 & 100.0 & 0.0 & 0.18 & 17.9 & 82.9 & 0.0 & 1.3 & 98.7 & 0.0 & 0.23 \\
\hline 8.055 & 26.9 & 78.6 & 0.0 & 3.4 & 96.6 & 0.0 & 0.72 & 6.9 & 88.0 & 9.9 & 0.2 & 25.6 & 74.2 & 0.15 \\
\hline 8.726 & 6.3 & 93.1 & 4.5 & 1.9 & 60.4 & 37.8 & 0.37 & 42.8 & 57.6 & 2.4 & 7.0 & 80.2 & 12.7 & 0.13 \\
\hline 9.397 & 32.7 & 65.2 & 7.3 & 3.4 & 27.5 & 69.1 & 0.48 & 29.4 & 68.2 & 3.4 & 2.3 & 45.4 & 52.3 & 0.00 \\
\hline 10.069 & 22.8 & 72.4 & 8.7 & 0.5 & 48.1 & 51.3 & 0.05 & 42.7 & 61.6 & 0.0 & 6.2 & 93.8 & 0.0 & 0.00 \\
\hline 10.740 & 29.4 & 63.1 & 7.8 & 3.3 & 48.0 & 48.7 & 0.00 & & & & & & & \\
\hline
\end{tabular}

x: contribuição em fluxo das populações, m: contribuição em massa e Av: correção por avermelhamento.
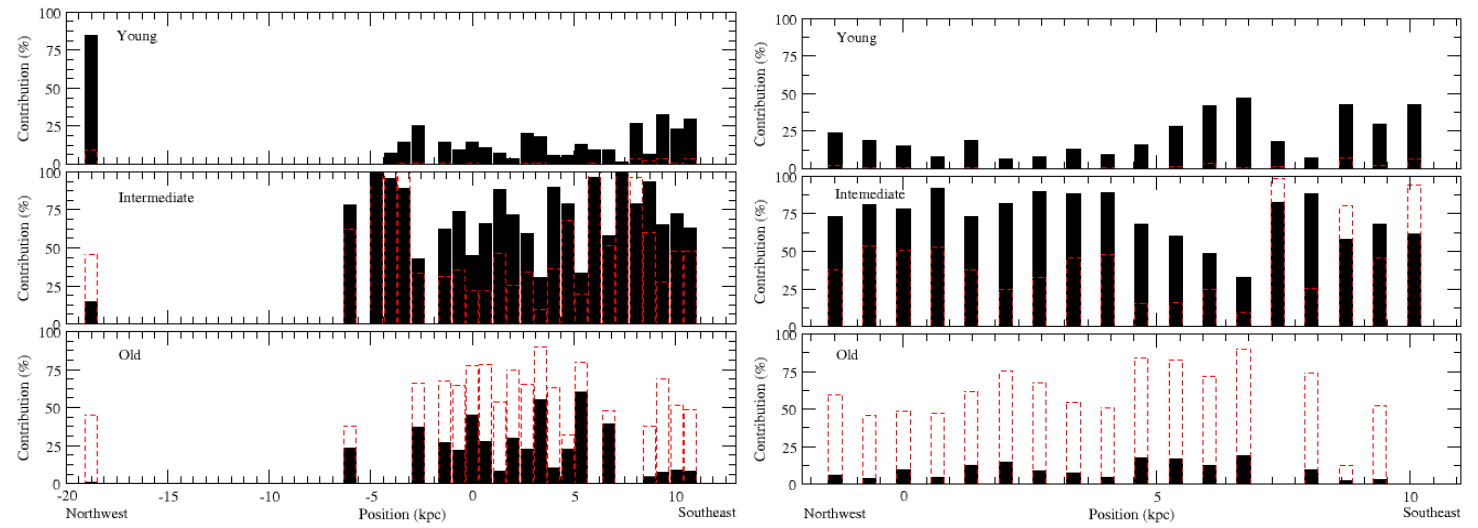

Figura 2 - Resultado da síntese espectral de população dada em função de fluxo (preto) e massa em vermelho como uma função da distância ao longo da posição de fenda $P A=292^{\circ}$

(Figura superior) e $\mathrm{PA}=325^{\circ}$ (Figura inferior).

\section{RESULTADOS}

Realizamos um estudo, utilizando síntese de população estelar, para investigar a história da população atual de estrelas nos sistemas: AM1054-325, AM1256-433, AM2030-303, AM2058-381 e AM2229-735, no qual apresentaremos somente os resultados obtidos da AM1256-433, 
mostrados na Figura 2, ao longo das posições de fenda observada $\mathrm{PA}=292^{\circ} \mathrm{e}$ $P A=325^{\circ}$. Segue, na Figura 3 , exemplo de espectro observado corrigido por avermelhamento (linha preta), o espectro sintético de população estelar sobreposto (linha vermelha), e o espectro de emissão puro (linha em rosa) para AM1256B.

A galáxia AM1256B apresenta uma variação espacial da distribuição de população sistemática ao longo das posições observadas, composta, principalmente, de população intermediária com idade mínima de 500 milhões de anos. Como podemos observar no resultado da posição $P A=292^{\circ}$, essa posição de fenda apresenta uma região localizada nas partes externas da direção Noroeste em 19 kpc, com um elevado percentual de população jovem (ver Figura 2 superior). Essa região inclui-se nas sete regiões $H_{\|}$luminosas, catalogadas por Ferreiro et al. (2008), no estudo desse objeto, com percentual de população estimada de $5,81 \times 10^{6}$ milhões de anos para essa região $H_{\|}$com, aproximadamente, a metade da idade jovem mínima de 100 milhões de anos assumida nesse trabalho. Nenhuma comparação pode ser feita devido à nossa região ser muito maior que uma típica região $H_{\|}$, mas confirma a predominância da existência de população jovem. Por outro lado, nas proximidades centrais e externas, esse componente é, predominantemente, intermediário, com um aumento significativo de população mais jovem do centro para as regiões externas.

Para a $\mathrm{PA}=325^{\circ}$, as contribuições das componentes são de predomínio de estrelas com idades intermediárias, somete nas regiões entre 6 e $7 \mathrm{kpc}$ o domínio é de população jovem, com baixo percentual de população velha, de $18,7 \%$. As mesmas contribuições pesadas em massa nas populações intermediárias, mas com baixa porcentagem de população jovem e aumento de população velha ao longo do raio.

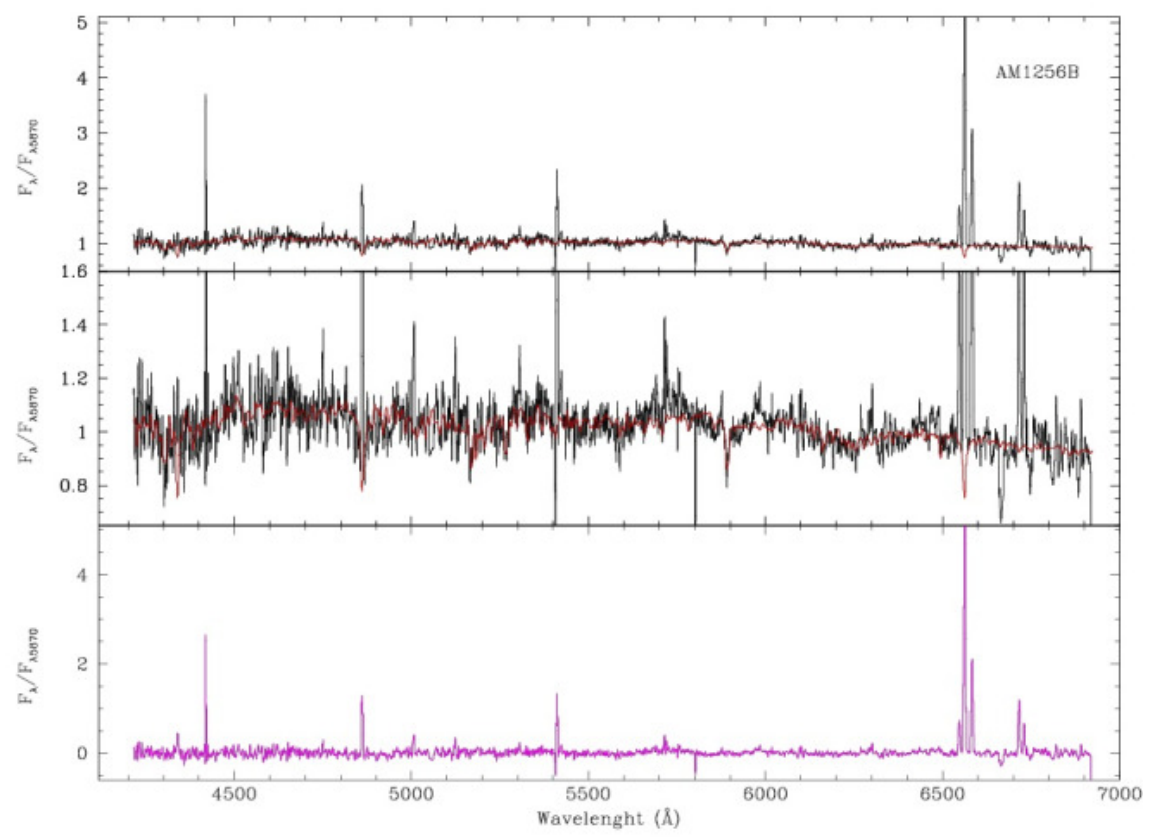

Figura 3 - Síntese de população estelar, obtida ao longo da posição de fenda da região do núcleo da $\mathrm{AM} 1256 \mathrm{~B}$, ao longo da posição $\mathrm{PA}=292^{\circ}$. 


\section{CONCLUSÃO}

Neste estudo, as galáxias AM1054A e AM2030B (ESO 463-IG 003 NED003) são dominadas por populações mais jovens, com idade de 500 bilhões de anos. Apenas a AM1054B apresentou um perfil de população velha ao longo de toda a sua região observada, as demais $\mathrm{AM} 1256 \mathrm{~B}$, AM2030A, AM2030B (ESO 463-IG 003 NED002), AM2058A, AM2058B apresentaram idades intermediárias decrescendo do centro para outras regiões. Basicamente, AM2229A apresenta uma população estelar composta de elementos heterogêneos ao longo de todas as posições observadas.

\section{AGRADECIMENTOS}

Deise A. Rosa, agradece ao departamento do IP\&D/UNIVAP e à FAPESP (Processo no 2011/08202-6), que proporcionam Bolsa Auxílio, para realização do curso de Doutorado em Física e Astronomia e aos orientadores, pela dedicação e paciência.

\section{REFERÊNCIAS BIBLIOGRÁFICAS}

BARNES, J. E.; HERNQUIST, L. Transformations of galaxies II gas dynamics in merging disk galaxies. Astrophysical Journal, 471, p. 115, 1996.

BRUZUAL, G.; CHARLOT, S. Stellar population synthesis at the resolution of 2003. Monthly Notices of the Royal Astronomical, v. 344, p. 1000-1028, 2003.

BUSHOUSE, H. A. Star-formation rates in the nuclei of violently interacting galaxies. Astronomical Journal, v. 91, p. 255-270, 1986.
CARDELLI, J. A.; CLAYTON, G. C.; MATHIS, J. S. The relationship between infrared, optical, and ultraviolet extinction. Astrophysical Journal, v. 345, p. 245-256, 1989.

CHABRIER, G. Galactic Stellar and Sub stellar Initial Mass Function. Astronomical Society of the Pacific, v. 115, p. 763-795, 2003.

CID FERNANDES, R. et al. Semi-empirical analysis of Sloan Digital Sky Survey galaxies - I. Spectral synthesis method. Monthly Notices of the Royal Astronomical Society, v. 358 , p. 363-378, 2004/2005.

DONZELLI, C. J.; PASTORIZA, M. G. Optical Spectroscopic Properties of a Sample of Interacting Galaxies. Astrophysical Journal Supplement, v. 111, p. 181, 1997.

FERREIRO, D. L.; PASTORIZA, M. G. Sample of minor merger of galaxies: Optical CCD surface photometry and HII region properties. Astronomy and Astrophysics, v. 428, p. 837-846, 2004.

FERREIRO, D. L.; PASTORIZA, M. G.; RICKES, M. Sample of minor merger of galaxies. II. Modelling HII region properties. Astronomy and Astrophysics, v. 481, p. 645649, 2008.

IONO, D.; YUN, M. S.; MIHOS, J. C. Radial Gas Flows in Colliding Galaxies: Connecting Simulations and Observations. Astrophysical Journal, v. 616, p. 199-220, 2004.

KENNICUTT, R. C. et al. The effects of interactions on spiral galaxies. II - Disk starformation rates. Astronomical Journal, v. 93, p. 1011-1023, 1987.

RICH, J. A.; KEWLEY, L. J.; DOPITA, M. A. Galaxy-wide Shocks in Late-merger Stage Luminous Infrared Galaxies. Astrophysical Journal, v. 734, p. 13-87, 2011.

Revista Univap - Edição Especial - revista.univap.br São José dos Campos-SP-Brasil, v. 19, n. 34, nov.2013. ISSN 2237-1753 
$\mathrm{RICH}$, J. A. et al. An Integral Field Study of Abundance Gradients in nearby Luminous Infrared Galaxies. Astrophysical Journal, v. 753, p. 5-20, 2012.

SOTO, K. T. et al. The Emission-line Spectra of Major Mergers: Evidence for Shocked Outflows. Astrophysical Journal, v. 757, p. 86, 2012.

SPRINGEL, V. The cosmological simulation code GADGET-2. Monthly Notices of the Royal Astronomical Society, v. 364, p. 11051134, 2005.
STRUCK, C. Galaxy collisions. Physics Reports, v. 321, p. 1-137, 1999.

VEILLEUX, S.; CECIL, G.; BLANDHAWTHORN, J. Galactic Winds. Annual Review of Astronomy and Astrophysics, v. 43, p. 769-826, 2005. 